\author{
Kuldip Raj, Charu Sharma and Suruchi Pandoh
}

\title{
MULTIPLICATION OPERATORS ON CESÀRO-ORLICZ SEQUENCE SPACES
}

\begin{abstract}
In this paper, we characterize the compact, invertible, Fredholm and closed range multiplication operators on Cesàro-Orlicz sequence spaces.

KEY WORDS: multiplication operator, Fredholm multiplication operator, invertible operator, compact operator, isometry, Cesàro sequence space, Cesàro-Orlicz sequence space.
\end{abstract}

AMS Mathematics Subject Classification: 47B38, 46A06.

\section{Introduction and preliminaries}

Cesàro sequence spaces $C e s_{p}, 1 \leq p<\infty$, appeared for the first time in 1968 as a problem of finding their duals [1]. Some basic properties of these spaces were studied in the early seventies by Shiue [23] and Leibowitz [13]. In 1974 Jagers [9] found the dual space of $\mathrm{Ces}_{p}$ (see also [12]). In the late nineties mathematicians became interested in geometric properties of these spaces. Cui and Płuciennik studied Local Uniform Nonsquareness [6] and Banach-Saks Property and Property $\beta$ [7], Cui and Hudzik studied Fixed Point Property [5] and obtained the Packing Constant [3].

An Orlicz function $\varphi:[0, \infty) \rightarrow[0, \infty)$ is a continuous, non-decreasing and convex such that $\varphi(0)=0, \varphi(x)>0$ for $x>0$ and $\varphi(x) \rightarrow \infty$ as $x \rightarrow \infty$. An Orlicz function is said to satisfy the $\delta_{2}-$ condition if their exists $K>0$ such that $\varphi(L x) \leq K L \varphi(x)$, for all $x \geq 0$ and for $L>1$. By $w$ we shall denote the space of all complex sequences. The Cesàro-Orlicz sequence space $\operatorname{Ces}_{\varphi}(\mathbb{N})$ is defined as

$$
\operatorname{Ces}_{\varphi}(\mathbb{N})=\left\{x=\left(x_{k}\right) \in w: \sum_{m=1}^{\infty} \varphi\left(\frac{1}{m} \sum_{k=1}^{m}\left|\lambda x_{k}\right|\right)<\infty\right\}
$$


The space $\operatorname{Ces}_{\varphi}(\mathbb{N})$ is a Banach space under the norm

$$
\|x\|=\inf \left\{\lambda>0: \sum_{m=1}^{\infty} \varphi\left(\frac{\frac{1}{m} \sum_{k=1}^{m}\left|x_{k}\right|}{\lambda}\right) \leq 1\right\} \quad(\text { see }[17]) .
$$

The Cesàro-Orlicz sequence spaces $\operatorname{Ces}_{\varphi}(\mathbb{N})$ appeared for the first time in 1988, when Lim and Lee [11] found their dual spaces. Recently, Cui et al. [4] obtained important properties of the spaces. In 2007 Maligranda et al. [14] showed that $\operatorname{Ces}_{\varphi}(\mathbb{N})$ is not B-convex, if $\varphi \in \delta_{2}$ and $\operatorname{Ces}_{\varphi}(\mathbb{N}) \neq\{0\}$. The extreme points and strong $U$-points of $\operatorname{Ces}_{\varphi}(\mathbb{N})$ have been characterized by Foralewski et al. in [8]. Although the spaces $\operatorname{Ces}_{\varphi}(\mathbb{N})$ have been studied by several mathematicians, some essential and basic properties remain still unknown but recently some of its properties have been discussed by Damian in $[10]$.

In the case when $\varphi(x)=|x|^{p},(p>1)$ the Cesàro-Orlicz sequence space $\operatorname{Ces}_{\varphi}(\mathbb{N})$ becomes the Cesàro sequence space $\mathrm{Ces}_{p}$.

Let $u: \mathbb{N} \rightarrow \mathbb{C}$ be a function such that $u . f \in \operatorname{Ces}_{\varphi}(\mathbb{N})$ for every $f \in \operatorname{Ces}_{\varphi}(\mathbb{N})$, then we can define a multiplication transformation $M_{u}$ : $\operatorname{Ces}_{\varphi}(\mathbb{N}) \rightarrow \operatorname{Ces}_{\varphi}(\mathbb{N})$ by

$$
M_{u} f=u f, \quad \forall f \in \operatorname{Ces}_{\varphi}(\mathbb{N}) .
$$

If $M_{u}$ is continuous, we call it a multiplication operator induced by $u$. These operators received considerable attention over the past several decades especially on $L^{p}$-spaces and Bergman spaces. From the recent literature available in Operator theory we find that multiplication operators are very much intimately connected with the composition operators as most of the properties of composition operators on $L^{p}$-spaces can be stated in terms of properties of multiplication operators. For example Singh and Kumar [21] proved that a composition operator on $L^{p}(X, \mathbb{C})$ is compact if and only if the multiplication operator $M_{u}$ is compact, where $u=\frac{d \mu T^{-1}}{d \mu}$, the Radon-Nikodym derivative of the measure $\mu T^{-1}$ with respect to the measure $\mu$. Infact the multiplication operators play an important role in the theory of Hilbert space operators. One of the main application is that every normal operator on a separable Hilbert space is unitarily equivalent to a multiplication operator. Moreover, multiplication operators has its roots in the spectral theory and is being pursued today in such guises as the theory of subnormal operators and the theory of Toeplitz operators. For more details on multiplication operators we refer to $([2],[18],[19],[20],[22],[24])$ and refrences therein. Moreover, Compact operators on sequence spaces have recently been studied by Mursaleen and Noman in $([15],[16])$. By $B\left(C e s_{\varphi}(\mathbb{N})\right)$ we denote the set of all bounded linear operators from $\operatorname{Ces}_{\varphi}(\mathbb{N})$ into itself. 
A bounded linear operator $A: E \rightarrow E$ (where $E$ is a Banach space) is called compact if $A\left(B_{1}\right)$ has compact closure, where $B_{1}$ denotes the closed unit ball of $E$.

A bounded linear operator $A: E \rightarrow E$ is called Fredholm if $A$ has closed range, $\operatorname{dim}(\operatorname{ker} A)$ and $\operatorname{co}-\operatorname{dim}(\operatorname{ran} A)$ are finite. The main purpose of this paper is to characterize the boundedness, compactness, closed range and Fredholmness of multiplication operators on Cesàro-Orlicz sequence spaces.

\section{Multiplication operators}

Theorem 1. Let $u: \mathbb{N} \rightarrow \mathbb{C}$ be a mapping. Then $M_{u}: \operatorname{Ces}_{\varphi}(\mathbb{N}) \rightarrow$ $C e s_{\varphi}(\mathbb{N})$ is bounded if and only if $u$ is a bounded function.

Proof. Suppose $u$ is a bounded function. Then there exists $M>0$ such that $\left|u_{n}\right| \leq M, \forall n \in \mathbb{N}$. Let $x \in \operatorname{Ces}_{\varphi}(\mathbb{N})$, we have

$$
\begin{aligned}
\left\|M_{u} x\right\| & =\sum_{m=1}^{\infty} \varphi\left(\frac{\frac{1}{m} \sum_{k=1}^{m}\left|(u x)_{k}\right|}{\lambda}\right) \\
& =\sum_{m=1}^{\infty} \varphi\left(\frac{\frac{1}{m} \sum_{k=1}^{m}\left|u_{k}\right|\left|x_{k}\right|}{\lambda}\right) \\
& \leq M \sum_{m=1}^{\infty} \varphi\left(\frac{\frac{1}{m} \sum_{k=1}^{m}\left|x_{k}\right|}{\lambda}\right) \\
& =M\|x\| .
\end{aligned}
$$

Thus, $\left\|M_{u} x\right\| \leq M\|x\|, \forall x \in C e s_{\varphi}(\mathbb{N})$ which implies that $M_{u}$ is a bounded operator.

Conversely, suppose that $M_{u}$ is a bounded operator. We show that $u$ is a bounded function. For, if $u$ is not a bounded function, then for every $n \in \mathbb{N}$, there exists some $p_{n} \in \mathbb{N}$ such that $\left|u\left(p_{n}\right)\right|>n$. Now $\left\|e^{p_{n}}\right\|=$ $\sum_{m=p_{n}}^{\infty} \frac{1}{m \lambda \varphi^{-1}(1)}$ and

$$
\begin{aligned}
\left\|M_{u} e^{p_{n}}\right\| & =\left(\sum_{m=p_{n}}^{\infty} \frac{\left|u_{m}\right|}{m \lambda \varphi^{-1}(1)}\right) \\
& >n\left(\sum_{m=p_{n}}^{\infty} \frac{1}{m \lambda \varphi^{-1}(1)}\right) \\
& =n\left\|e^{p_{n}}\right\| .
\end{aligned}
$$

This shows that $M_{u}$ is not a bounded operator. Hence, $u$ must be a bounded function. 
Theorem 2. $M_{u}$ is an isometry if and only if $\left|u_{n}\right|=1, \forall n \in \mathbb{N}$.

Proof. Suppose first that $\left|u_{n}\right|=1, \forall n \in \mathbb{N}$. Then

$$
\begin{aligned}
\left\|M_{u} x\right\| & =\sum_{m=1}^{\infty} \varphi\left(\frac{\frac{1}{m} \sum_{k=1}^{m}\left|u_{k} x_{k}\right|}{\lambda}\right) \\
& =\sum_{m=1}^{\infty} \varphi\left(\frac{\frac{1}{m} \sum_{k=1}^{m}\left|x_{k}\right|}{\lambda}\right) \\
& =\|x\| .
\end{aligned}
$$

Therefore, $\left\|M_{u} x\right\|=\|x\|, \forall x \in \operatorname{Ces}_{\varphi}(\mathbb{N})$ and hence $M_{u}$ is an isometry.

Conversely, suppose that $\left|u_{n}\right| \neq 1$ for some $n=n_{0}$. Then $\left\|e^{n_{0}}\right\|=$ $\sum_{m=n_{0}}^{\infty} \frac{1}{m \lambda \varphi^{-1}(1)}$. Suppose $\left|u_{n_{0}}\right|>1$. Then

$$
\begin{aligned}
\left\|M_{u} e^{n_{0}}\right\| & =\left(\sum_{m=n_{0}}^{\infty} \frac{\left|u_{n_{0}}\right|}{m \lambda \varphi^{-1}(1)}\right) \\
& >\sum_{m=n_{0}}^{\infty} \frac{1}{m \lambda \varphi^{-1}(1)} \\
& =\left\|e^{n_{0}}\right\| .
\end{aligned}
$$

Similarly if $\left|u_{n_{0}}\right|<1$, then we can show that $\left\|M_{u} e^{n_{0}}\right\|<\left\|e^{n_{0}}\right\|$. In both cases, when $\left|u_{n_{0}}\right|<1$ or $\left|u_{n_{0}}\right|>1$, we get contradiction. Hence, $\left|u_{n}\right|=$ $1 \forall n \in \mathbb{N}$.

\section{Compact multiplication operators}

Theorem 3. Let $M_{u} \in B\left(\operatorname{Ces}_{\varphi}(\mathbb{N})\right)$. Then $M_{u}$ is a compact operator if and only if $u(n) \rightarrow 0$ as $n \rightarrow \infty$.

Proof. We first assume that $M_{u}$ is a compact operator. We show that $u(n) \rightarrow 0$ as $n \rightarrow \infty$. For if this were not true, then there exists $\epsilon>0$ such that the set $A_{\epsilon}=\left\{k \in \mathbb{N}:\left|u_{k}\right| \geq \epsilon\right\}$ is an infinite set. Let $p_{1}, p_{2}, \ldots, p_{n}, .$. be in $A_{\epsilon}$. Then $\left\{e^{p_{n}}: p_{n} \in A_{\epsilon}\right\}$ is an infinite bounded set in $\operatorname{Ces}_{\varphi}(\mathbb{N})$. Consider

$$
\begin{aligned}
\left\|M_{u} e^{p_{n}}-M_{u} e^{p_{s}}\right\| & =\sum_{m=1}^{\infty} \varphi\left(\frac{\frac{1}{m} \sum_{k=1}^{m}\left|u(k) e^{p_{n}}(k)-u(k) e^{p_{s}}(k)\right|}{\lambda}\right) \\
& =\sum_{m=1}^{\infty} \varphi\left(\frac{\frac{1}{m} \sum_{k=1}^{m}|u(k)|\left|e^{p_{n}}(k)-e^{p_{s}}(k)\right|}{\lambda}\right) \\
& \geq \epsilon\left\|e^{p_{n}}-e^{p_{s}}\right\|, \forall p_{n}, p_{s} \in A_{\epsilon} .
\end{aligned}
$$


This proves that $\left\{e^{p_{n}}: p_{n} \in A_{\epsilon}\right\}$ is a bounded sequence which cannot have a convergent subsequence under $M_{u}$. This shows that $M_{u}$ cannot be a compact operator, which is a contradiction. Hence, $u(n) \rightarrow 0$ as $n \rightarrow \infty$.

Conversely, suppose $u(n) \rightarrow 0$ as $n \rightarrow \infty$. Then for every $\epsilon>0$, the set $A_{\epsilon}=\{n \in \mathbb{N}:|u(n)| \geq \epsilon\}$ is a finite set. Then $\operatorname{Ces}_{\varphi}\left(A_{\epsilon}\right)$ is a finite dimensional space for each $\epsilon>0$. Therefore, $M_{u} \mid \operatorname{Ces}_{\varphi}\left(A_{\epsilon}\right)$ is a compact operator. For each $n \in \mathbb{N}$, define $u_{n}: \mathbb{N} \rightarrow \mathbb{C}$ by

$$
u_{n}(m)= \begin{cases}u(m), & \forall m \in A_{\frac{1}{n}} \\ 0, & \forall m \notin A_{\frac{1}{n}}\end{cases}
$$

Clearly, $M_{u_{n}}$ is a compact operator as the space $\operatorname{Ces}_{\varphi}\left(A_{\frac{1}{n}}\right)$ is finite dimensional for each $n \in \mathbb{N}$. Now

$$
\begin{aligned}
\left\|\left(M_{u_{n}}-M_{u}\right) x\right\|= & \sum_{m=1}^{\infty} \varphi\left(\frac{\frac{1}{m} \sum_{k=1}^{m}\left|u_{n}(k) x_{k}-u(k) x_{k}\right|}{\lambda}\right) \\
= & \sum_{m \in A_{\frac{1}{n}}}^{\infty} \varphi\left(\frac{\frac{1}{m} \sum_{k=1}^{m}\left|u_{n}(k) x_{k}-u(k) x_{k}\right|}{\lambda}\right) \\
& +\sum_{m \in A_{\frac{1}{n}}^{\prime}}^{\infty} \varphi\left(\frac{\frac{1}{m} \sum_{k=1}^{m}\left|u_{n}(k) x_{k}-u(k) x_{k}\right|}{\lambda}\right) \\
= & \sum_{m \in A_{\frac{1}{n}}^{\prime}}^{\infty} \varphi\left(\frac{\frac{1}{m} \sum_{k=1}^{m}\left|u(k) x_{k}\right|}{\lambda}\right) \\
< & \frac{1}{n} \sum_{m \in A_{\frac{1}{n}}^{\prime}}^{\infty} \varphi\left(\frac{\frac{1}{m} \sum_{k=1}^{m}\left|x_{k}\right|}{\lambda}\right) \\
\leq & \frac{1}{n}\|x\|^{\infty}
\end{aligned}
$$

or $\left\|\left(M_{u_{n}}-M_{u}\right)(x)\right\| \leq \frac{1}{n}\|x\|$. This proves that $\left\|\left(M_{u_{n}}-M_{u}\right)\right\| \leq \frac{1}{n}$ and that $M_{u}$ is a limit of compact operators and hence, $M_{u}$ is a compact operator.

Theorem 4. Let $M_{u} \in B\left(\operatorname{Ces}_{\varphi}(\mathbb{N})\right)$. Then $M_{u}$ has closed range if and only if $u$ is bounded away from zero on $\mathbb{N} \backslash$ keru $=S$.

Proof. Suppose $u$ is bounded away from zero on $S$. Then there exists $\epsilon>0$ such that $\left|u_{n}\right| \geq \epsilon \forall n \in \mathbb{N} \backslash$ keru. We have to prove that $\operatorname{ran} M_{u}$ 
is closed. Let $z$ be a limit point of $\operatorname{ran} M_{u}$. Then there exists a sequence $M_{u} x^{n} \rightarrow z$. Clearly, the sequence $\left\{M_{u} x^{n}\right\}$ is a Cauchy sequence. Now,

$$
\begin{aligned}
\left\|M_{u} x^{n}-M_{u} x^{m}\right\| & =\sum_{m=1}^{\infty} \varphi\left(\frac{\frac{1}{m} \sum_{k=1}^{m}\left|u_{k} x_{k}^{n}-u_{k} x_{k}^{m}\right|}{\lambda}\right) \\
& =\sum_{m=1}^{\infty} \varphi\left(\frac{\frac{1}{m} \sum_{k=1}^{m}\left|u_{k}\right|\left|x_{k}^{n}-x_{k}^{m}\right|}{\lambda}\right) \\
& \geq \epsilon \sum_{m=1}^{\infty} \varphi\left(\frac{\frac{1}{m} \sum_{k=1}^{m}\left|x_{k}^{n}-x_{k}^{m}\right|}{\lambda}\right) \\
& =\epsilon \sum_{m=1}^{\infty} \varphi\left(\frac{\frac{1}{m} \sum_{k=1}^{m}\left|\tilde{x_{k}^{n}}-\tilde{x_{k}^{m}}\right|}{\lambda}\right) \\
& =\epsilon\left\|\tilde{x^{n}}-\tilde{x^{m}}\right\|,
\end{aligned}
$$

where

$$
\tilde{x_{k}^{n}}= \begin{cases}x_{k}^{n}, & \text { if } k \in S \\ 0, & \text { if } k \notin S .\end{cases}
$$

This proves that $\left\{\tilde{x_{n}}\right\}$ is a Cauchy sequence in $\operatorname{Ces}_{\varphi}(\mathbb{N})$. But $\operatorname{Ces}_{\varphi}(\mathbb{N})$ is complete. Therefore, there exists $x \in \operatorname{Ces}_{\varphi}(\mathbb{N})$ such that $\tilde{x}^{n} \rightarrow x$ as $n \rightarrow \infty$. Inview of continuity of $M_{u}, M_{u} \tilde{x^{n}} \rightarrow M_{u} x$. But $M_{u} x^{n}=M_{u} \tilde{x^{n}} \rightarrow z$. Therefore, $M_{u} x=z$. Hence, $z \in \operatorname{ran} M_{u}$. This proves that $M_{u}$ has closed range.

Conversely, suppose that $M_{u}$ has closed range. Then $M_{u}$ is bounded away from zero on $\left(\operatorname{ker} M_{u}\right)^{\perp}=\operatorname{Ces}_{\varphi}(\mathbb{N} \backslash$ keru $)$. That is, there exists $\epsilon>0$ such that

$$
\left\|M_{u} x\right\| \geq \epsilon\|x\| \forall x \in C e s_{\varphi}(\mathbb{N} \backslash \text { keru }) .
$$

Let $G=\left\{k \in \mathbb{N} \backslash k e r u:\left|u_{k}\right|<\frac{\epsilon}{2}\right\}$. If $G \neq \phi$, then for $n_{0} \in G$, we have

$$
\begin{aligned}
\left\|M_{u} e^{n_{0}}\right\| & =\sum_{m=1}^{\infty} \varphi\left(\frac{\frac{1}{m} \sum_{k=1}^{m}\left|u(k) e^{n_{0}}(k)\right|}{\lambda}\right) \\
& =\sum_{m=n_{0}}^{\infty}\left(\frac{\left|u\left(n_{0}\right)\right|}{m \lambda \varphi^{-1}(1)}\right) \\
& <\epsilon \sum_{m=n_{0}}^{\infty} \frac{1}{m \lambda \varphi^{-1}(1)} \\
& =\epsilon\left\|e^{n_{0}}\right\| .
\end{aligned}
$$

That is, $\left\|M_{u} e^{n_{0}}\right\|<\epsilon\left\|e^{n_{0}}\right\|$ which contradicts (1). Hence, $G=\phi$ so that $\left|u_{k}\right| \geq \epsilon, \forall k \in \mathbb{N} \backslash k e r u$. This proves the theorem. 


\section{Invertible and Fredholm multiplication operators}

Theorem 5. Let $u: \mathbb{N} \rightarrow \mathbb{C}$ be a mapping. Then $M_{u}: \operatorname{Ces}_{\varphi}(\mathbb{N}) \rightarrow$ $\mathrm{Ces}_{\varphi}(\mathbb{N})$ is invertible if and only if there exist $m>0$ and $M>0$ such that $m<u_{n}<M, \forall n \in \mathbb{N}$.

Proof. Suppose that the condition is true. Define $\gamma: \mathbb{N} \rightarrow \mathbb{C}$ by $\gamma_{n}=\frac{1}{u_{n}}$. Then $M_{u}$ and $M_{\gamma}$ are bounded linear operators in view of Theorem 1. Also $M_{u} \cdot M_{\gamma}=M_{\gamma} \cdot M_{u}=I$. Hence, $M_{\gamma}$ is the inverse of $M_{u}$.

Conversely, suppose that $M_{u}$ is invertible. Then $\operatorname{ran}_{u}=\operatorname{Ces}_{\varphi}(\mathbb{N})$. Therefore, $\operatorname{ran} M_{u}$ is closed. Hence, by Theorem 4, there exists $\epsilon>0$ such that $\left|u_{n}\right| \geq \epsilon \forall n \in \mathbb{N} \backslash$ keru. Now keru $=\phi$, otherwise $u_{n_{0}}=0$, for some $n_{0} \in \mathbb{N}$, in which case $e^{n_{0}} \in \operatorname{ker} M_{u}$ which is a contradiction, since $\operatorname{ker} M_{u}$ is trivial. Hence, $\left|u_{n}\right| \geq \epsilon \forall n \in \mathbb{N}$. Since $M_{u}$ is bounded, so by Theorem 1, there exists $M>0$ such that $\left|u_{n}\right| \leq M, \forall n \in \mathbb{N}$. Thus, we have proved that $\epsilon \leq\left|u_{n}\right| \leq M, \forall n \in \mathbb{N}$.

Theorem 6. Let $M_{u}: \operatorname{Ces}_{\varphi}(\mathbb{N}) \rightarrow \operatorname{Ces}_{\varphi}(\mathbb{N})$ be a bounded operator. Then $M_{u}$ is Fredholm operator if and only if

(i) keru is a finite subset of $\mathbb{N}$.

(ii) $\left|u_{n}\right| \geq \epsilon, \forall n \in \mathbb{N} \backslash$ keru.

Proof. Suppose $M_{u}$ is Fredholm. If keru is an infinite subset of $\mathbb{N}$, then $e^{n} \in \operatorname{ker} M_{u} \forall n \in$ keru. But $e^{n}$ 's are linearly independent, which shows that $\operatorname{ker} M_{u}$ is an infinite dimensional which is a contradiction. Hence, keru must be a finite subset of $\mathbb{N}$. The condition (ii) follows from Theorem 4 .

Conversely, If the conditions $(i)$ and $(i i)$ are true, then we prove that $M_{u}$ is Fredholm. In view of Theorem 4, the condition (ii) implies that $M_{u}$ has closed range. The condition $(i)$ implies that $\operatorname{ker} M_{u}$ and $\operatorname{ker} M_{u}^{\star}$ are finite dimensional. This proves that $M_{u}$ is Fredholm.

\section{References}

[1] Programma van Jaarlijkse PriJsvragen, (Annual Problem Section), Nieuw Arch. Wiskd., 16(1968), 47-51.

[2] Abrahmse M.B., Multiplication operators, Lecture Notes in Mathematics, 693 Springer Verlag (1978), 17-36.

[3] Cui Y., Hudzik H., Packing constant for Cesàro sequence spaces, Nonlinear Anal., 47(2001), 2695-2702.

[4] Cui Y., Hudzik H., Petrot N., Szymaszkiewicz A., Basic topological and geometric properties of Cesàro-Orlicz spaces, Proc. Indian Acad. Sci. Math. Sci., 115(2005), 461-476.

[5] Cui Y., Hudzik H., Some geometric properties related to fixed point theory in Cesàro spaces, Collect. Math., 50(1999), 277-288. 
[6] Cui Y., R.PŁuciennik, Local uniform nonsquareness in Cesàro sequence spaces, Comment. Math. Prace Mat., 37(1997), 47-58.

[7] Cui Y., R.PŁuciennik, Banach-Saks property and property $\beta$ in Cesàro sequence spaces, Southeast Asian Bull. Math., 24(2000), 201-210.

[8] Foralewski P., Hudzik H., Szymaszkiewicz A., Local rotundity structure of Cesàro-Orlicz spaces, J. Math. Anal. Appl., 345(2008), 410-419.

[9] Jagers A.A., A note on Cesàro sequence spaces, Nieuw Arch. Wiskund, 22 (1974), 113-124.

[10] Kubiak D.M., A note on Cesàro-Orlicz spaces, J. Math. Anal. Appl., 349 (2009), 291-296.

[11] Lim S.K., LeE P.Y., An Orlicz extension of Cesàro sequence spaces, Comment. Math. Prace Mat., 28(1988), 117-128.

[12] LeE P.Y., Cesàro sequence spaces, Math. Chronicle, New Zealand, 13(1984), $29-45$.

[13] Leibowitz G.M., A note on Cesàro sequence spaces, Tamkang J. Math., 2 (1971), 151-157.

[14] Maligranda L., Petrot N., Suantai S., On the james constants and B-convexity of Cesàro and Cesàro-Orlicz sequence spaces, J. Math. Anal. Appl., 326(2007), 312-326.

[15] Mursaleen M., Noman A.K., Compactness by the Hausdorff measure of noncompactness, Nonlinear Anal., 73(2010), 2541-2557.

[16] Mursaleen M., Noman A.K., Compactness of matrix operators on some new difference sequence spaces, Linear Algebra Appl., 436(2012), 41-52.

[17] Petrot N. Suantai S., Some geometric properties in Orlicz-Cesàro spaces, Science Asia, 31(2005), 173-177.

[18] Raj K., Sharma S.K., Kumar A., Multiplication operator on MusielakOrlicz spaces of Bochner type, J. Adv. Stud. Topol., 3(2012), 1-7.

[19] Sharma A., RaJ K., Sharma S.K., Products of multiplication composition and differentiation operators from $H^{\infty}$ to weighted Bloch spaces, Indian J. Math., 54(2012), 159-179.

[20] Singh R.K., Manhas J.S., Composition Operators on Function Spaces, North Holland, 1993.

[21] Singh R.K., Kumar A., Multiplication operators with closed range, Bull. Aust. Math. Soc., 16(1977), 247-252.

[22] Singh R.K., Manhas J.S., Multiplication operators and composition operators with closed ranges, Bull. Aust. Math. Soc., 16(1977), 247-252.

[23] Shiue J.S., On the Cesàro sequence spaces, Tamkang J. Math., 1(1970), $19-25$.

[24] Takagi H., Yokouchi K., Multiplication and composition operators between two $L^{p}$-spaces, Contemp. Math., 232(1999), 321-338.

\author{
KulDiP RAJ \\ Department of Mathematics \\ Shri Mata Vaishno Devi University \\ KATRA-182320, J \& K, InDiA \\ e-mail: kuldipraj68@gmail.com
}




\author{
Charu Sharma \\ Department of Mathematics \\ Shri Mata Vaishno Devi University \\ KATRA-182320, J \& K, IndiA \\ e-mail: charu145.cs@gmail.com \\ SuRUChI PANDOH \\ Department of Mathematics \\ Shri Mata Vaishno Devi University \\ KATRA-182320, J \& K, IndiA \\ e-mail: suruchi.pandoh87@gmail.com
}

Received on 07.09.2016 and, in revised form, on 24.11.2016. 